\title{
Helminth Excreted/Secreted Antigens Repress Expression of LPS-Induced Let-7i but Not miR-146a and miR-155 in Human Dendritic Cells
}

\author{
Luis I. Terrazas, ${ }^{1}$ Fausto Sánchez-Muñoz, ${ }^{2}$ \\ Magaly Pérez-Miranda, ${ }^{2}$ Ana M. Mejía-Domínguez, ${ }^{3}$ Yadira Ledesma-Soto, ${ }^{1}$ \\ Rafael Bojalil, ${ }^{2,4}$ and Lorena Gómez-García ${ }^{2}$ \\ ${ }^{1}$ Biomedicine Unit, Facultad de Estudios Superiores Iztacala, UNAM, Avenida de los Barrios No. 1 Col. Los Reyes Iztacala, \\ 54090 Tlalnepantla, MEX, Mexico \\ ${ }^{2}$ Department of Immunology, Instituto Nacional de Cardiología Ignacio Chávez, Juan Badiano No. 1 Col. Sección XVI, \\ 140080 Tlalpan, DF, Mexico \\ ${ }^{3}$ Blood Bank, Instituto Nacional de Cardiología Ignacio Chávez, Juan Badiano No. 1 Col. Sección XVI, 140080 Tlalpan, DF, Mexico \\ ${ }^{4}$ Department of Health Care, Universidad Autónoma Metropolitana Xochimilco, Calzada del Hueso No. 1100 Col. Villa Quietud, \\ 04960 Coyoacán, DF, Mexico
}

Correspondence should be addressed to Lorena Gómez-García; lorena.gomez@cardiologia.org.mx

Received 7 August 2012; Accepted 1 October 2012

Academic Editor: Miriam Rodríguez-Sosa

Copyright (C) 2013 Luis I. Terrazas et al. This is an open access article distributed under the Creative Commons Attribution License, which permits unrestricted use, distribution, and reproduction in any medium, provided the original work is properly cited.

\begin{abstract}
MicroRNAs have emerged as key regulators of immune responses. They influence immune cells' function and probably the outcome of several infections. Currently, it is largely unknown if helminth parasites and their antigens modify host microRNAs expression. The aim of this study was to explore if excreted/secreted antigens of Taenia crassiceps regulate LPS-induced miRNAs expression in human Dendritic Cells. We found that these antigens repressed LPS-let-7i induction but not mir-146a or mir-155 and this correlates with a diminished inflammatory response. This let-7i downregulation in Dendritic Cells constitutes a novel feature of the modulatory activity that helminth-derived antigens exert on their host.
\end{abstract}

\section{Introduction}

MicroRNAs (miRNAs) are small ( 23 nucleotides) noncoding RNAs that negatively regulate protein-coding gene expression mainly via down-regulation of mRNA levels and/or translational suppression [1]. In the last years, several reports have linked miRNAs to multiple and essential functions in the immune system. These molecules have emerged as important regulators of the development and differentiation of $T$ and $B$ lymphocytes and dendritic cells (DCs) [2-4], as modulators of inflammation [5], of balance between Th1 and Th2 responses [6], and of antibody production [7] amongst other functions. Besides their physiological role, miRNAs participate in pathological aspects of the immune response; for example, these molecules may be important mediators in cancer and autoimmunity [8] but participate as well in the control of viral infections [9]. The response of host miRNAs in parasitic infections is largely unknown. The few reports that have addressed the influence of parasites upon host-derived miRNAs have focused on protozoan infections $[10,11]$ while the role of helminth parasites and their antigens as possible modulators of such miRNAs is widely unexplored.

Dendritic Cells (DCs) dictate immune responses through the different signals derived from them such as secreted cytokines, chemokines, and also costimulatory molecules. Once an immature DC faces stimuli, either exogenous or endogenous, this cell will undergo an intracellular process that ultimately will render it capability of supporting the different types of immune responses [13]. DCs exposed to lipopolysaccharide (LPS) are cells that suffer a typical maturation process with secretion of inflammatory cytokines and 
chemokines mainly IL-12, TNF, IL-6, RANTES, MCP1 among others, and an upregulation of certain membrane molecules. Altogether, these changes confer DCs with the capability to induce the appropriate adaptive immune responses [14, 15]. Recently, it has been described that some miRNAs are upregulated and may participate in these dendritic cell's maturation/activation events $[16,17]$. Specifically, miR-146a, miR-155, and let-7i are microRNAs that are modulated positively when DCs are exposed to maturation agents including LPS, TNF, and IFN- $\gamma[18,19]$ and seem to be related to the activation events triggered in DCs by such stimuli including the expression of costimulatory molecules, secretion of proinflammatory cytokines, and even induction of apoptosis [16-18]. Although there is one report showing that chronic ascariasis and trichuriasis modify the expression of miRNA let-7d in peripheral-blood mononuclear cells (PBMCs) [20], the impact of helminthes and their antigens on DCs-derived miRNAs and its possible consequences for the function of these cells has not been addressed to date.

We recently demonstrated that some properties of human DCs can be affected by their exposure to the excreted/ secreted antigens derived from the cysticerci of Taenia crassiceps (TcES) [12]. These cells were characterized by an immature phenotype with low expression of the molecules CD80, CD86, and CD83 and were capable of secreting the regulatory cytokine IL-10 but not any of the inflammatory cytokines tested. More importantly, DCs exposed to TcES showed impaired maturation/activation to subsequent LPS stimulation, where both expression of costimulation molecules and secretion of proinflammatory cytokines were significantly diminished, indicating the anti-inflammatory effects of TcES upon this cell type [12]. Since TcES affects events related with the activation of DCs by LPS, here we explored if these parasite antigens modify the expression in human DCs of the microRNAs miR-146a, miR-155, and let7i, which, as stated earlier, seem to play a critical role in the classical maturation and activation processes induced by LPS in these cells.

\section{Methods}

2.1. Taenia crassiceps Excreted/Secreted Antigens. Metacestodes of Taenia crassiceps were harvested from the peritoneal cavity of female Balb/c mice after 2-4 months of infection. This was done under a laminar flow chamber and sterile $1 \mathrm{X}$ PBS was used. The cysticerci were washed four times with 1X PBS and maintained in culture in $1 \mathrm{X}$ PBS at $37^{\circ} \mathrm{C}$ for $24 \mathrm{~h}$. TcES were recovered from the supernatant and centrifuged for $10 \mathrm{~min}$ at $1000 \mathrm{~g}$ using LPS-free filters. This fraction was concentrated using $50 \mathrm{kDa}$ Amicon Ultra Filter (Millipore). Concentrations of different lots range between 400 and $920 \mathrm{ug} / \mathrm{mL}$. Samples were stored at $-70^{\circ} \mathrm{C}$ until further use.

2.2. Monocyte-Derived Dendritic Cells. Human peripheral blood mononuclear cells (PBMCs) were obtained from buffy coats of 12 healthy blood donors from the Instituto Nacional de Cardiología Ignacio Chávez’s Blood Bank. Informed consent was obtained for the use of blood samples according to the declaration of Helsinki and the local scientific and ethics committees approved the protocol. PBMCs were isolated by Ficoll-gradient centrifugation (GE Healthcare), analyzed in a Coulter AcT for cellular types (Beckman Coulter), and $3 \times 10^{6}$ monocytes were left to adhere in 6 -well culture plates for $2 \mathrm{~h}$. After this period, nonadherent cells were washed away and adherent cells were cultured in RPMI medium supplemented with $10 \%$ SFB and penicillin/streptomycin in presence of 400 $\mathrm{U} / \mathrm{mL}$ of IL- 4 and $800 \mathrm{U} / \mathrm{mL}$ of GM-CSF during 6 days with replacement of medium and cytokines at day 3. At day 6 nonadherent cells were recovered and placed for $24 \mathrm{~h}$ in fresh medium. At this point we determined by flow cytometry the percentage of CD11c+ cells and for all experiments this was $\geq 80 \%$. Cells were challenged with $20 \mathrm{ug} / \mathrm{mL}$ TcES, $1 \mathrm{ug} / \mathrm{mL}$ LPS, or a combination of them for 3 or $24 \mathrm{~h}$. Control cells received RPMI.

2.3. QRT-PCR for Mature MicroRNAs. To asses mature microRNA expression, we used two-step qRT-PCR, with TaqMan microRNA assays (Applied Biosystems). For microRNA cDNA synthesis, a $15 \mu \mathrm{L}$ reaction volume, composed of $1.5 \mu \mathrm{L}$ of Buffer (10x), $0.15 \mu \mathrm{L} 100 \mathrm{mM}$ dNTPs $(100 \mathrm{mM}), 1.0 \mu \mathrm{L}$ reverse transcriptase, $0.19 \mu \mathrm{L}$ RNAse inhibitor $(20 \mathrm{U} / \mu \mathrm{L})$, and $3.0 \mu \mathrm{L}$ of each specific microRNA primer, was mixed with $10 \mathrm{ng}$ of total RNA. RT reaction was incubated 30 minutes of $16^{\circ} \mathrm{C}, 30$ minutes of $42^{\circ} \mathrm{C}$, and 5 minutes of $85^{\circ} \mathrm{C}$. Duplicate real-time PCR reactions were performed in a Roche LigthCycler 2.0. Reaction mix was composed of 1x LigthCycler TaqMan Master (Roche), 1X of each specific microRNA probe, and $2.5 \mathrm{uL}$ of specific microRNA cDNA (diluted $1: 3$ ). These were followed by 10 minutes $95^{\circ} \mathrm{C}$ for preincubation, 40 cycles of amplification program consisting of $95^{\circ} \mathrm{C} 10 \mathrm{sec}, 60^{\circ} \mathrm{C} 40 \mathrm{sec}$, and $72^{\circ} \mathrm{C}$ $5 \mathrm{sec}$ (fluorescence acquisition). To asses possible bias for reference gene selection U6 and let7a were used as reference genes, and relative quantification was calculated by the formula 2-(Cp microRNA target-Cp U6 or let7a). All microRNA assays were tested for reproducibility and linearity (PCR efficiency was between 1.9 and 2.0 for all assays).

2.4. Quantification of Cytokine Production. Supernatants were recovered after $3 \mathrm{~h}$ and $24 \mathrm{~h}$ stimulation period and production of the cytokines TNF, IL-6, IL-12, IL-10 (Peprotech), and MCP-1 (R\&D) was measured by ELISA kits in the supernatants of DCs cultures. For the analysis of cytokine mRNAs levels cells were recovered from cultures $3 \mathrm{~h}$ after stimulation.

2.5. Statistics. Comparisons were performed by the MannWhitney Wilcoxon test for cytokine comparisons and Kruskal-Wallis and Dunn's post hoc test for miRNAs expression. Significance was set on a $P$ value $<0.05$. All analyses were performed with the GraphPad Prism v. 5 statistical software.

\section{Results and Discussion}

3.1. TcES Dampen the Inflammatory Activity of LPS in Human DCs. LPS activation of DCs induces their maturation and secretion of pro-inflammatory cytokines and chemokines 
$3 \mathrm{~h}$
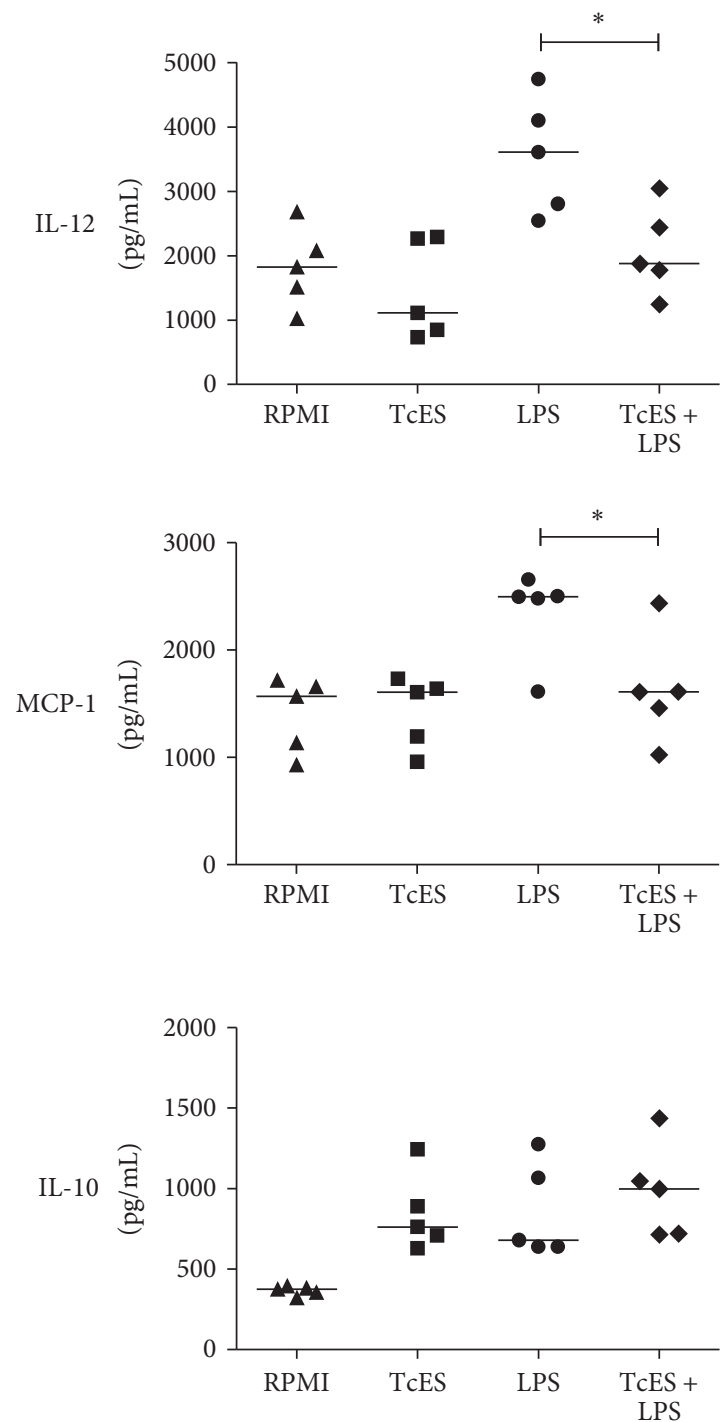

$24 \mathrm{~h}$
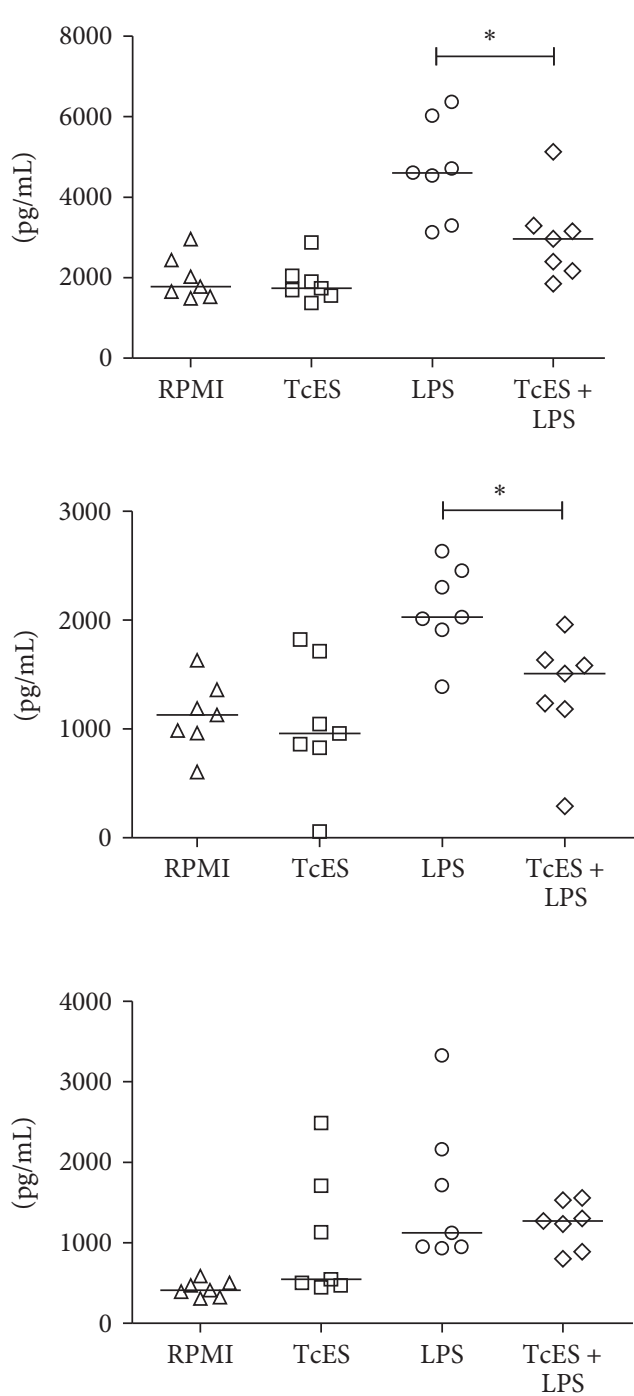

FIGURE 1: TcES modify the secretion of cytokines by human DCs activated with LPS. Human peripheral blood mononuclear cells (PBMCs) were obtained from buffy coats of 12 healthy blood donors. Informed consent was obtained for the use of blood samples according to the declaration of Helsinki and the local scientific and ethics committees approved the protocol. Monocyte-derived DCs were cultured for six days in the presence of IL-4 and GM-CSF [12]. Cells were challenged with $20 \mathrm{ug} / \mathrm{mL}$ TcES, $1 \mathrm{ug} / \mathrm{mL} \mathrm{LPS}$, or a combination of them for $3 \mathrm{~h}$ or $24 \mathrm{~h}$. Control cells received RPMI. Cells and supernatants were collected and cytokine response was measured by ELISA kits (Peprotech and $\mathrm{R} \& \mathrm{D})$. The data is shown as scattered plots and medians of twelve independent experiments ( $n=5$ for $3 \mathrm{~h}$ and $n=7$ for $24 \mathrm{~h}$ ). Comparisons were performed by the Mann-Whitney test. ${ }^{*} P<0.05$ LPS versus LPS/TcES. All analyses were performed with the GraphPad Prism v. 5 statistical software.

such as IL-12, TNF, IL-6, MCP-1 among others [11, 13]. We demonstrated previously that TcES does not induce secretion of the pro-inflammatory cytokines IL-12, IL-1 $\beta$, TNF, or IL- 6 but contrary it upregulates the secretion of IL-10. More importantly, these cysticerci-derived antigens interfered with the LPS-activation of DCs, downmodulating their maturation, and reversing the secretion of all the pro-inflammatory cytokines tested but not the one of regulatory cytokine IL-10 [12].

We tested if the phenomenon of modulation of TcES upon LPS-induced cytokine response was observed in the monocyte-derived DCs of the donors used for this study. At day six of culture, DCs received TcES, LPS, or a combination of both for $3 \mathrm{~h}$ and $24 \mathrm{~h}$, and supernatants were recovered. As expected, we found that unlike LPS, TcES did not induce secretion of the inflammatory molecules IL-12 and MCP-1 (Figure 1) but instead, these antigens were capable of downmodulating such response to LPS both at $3 \mathrm{~h}$ or $24 \mathrm{~h}$ after stimulation (Figure 1). Interestingly, secretion of the regulatory cytokine IL-10 was induced similarly by TcES, LPS, and the combination of both (Figure 1). Altogether, these results indicate that these helminth antigens promote human DCs 
to acquire an anti-inflammatory phenotype while dampening the inflammatory response induced by LPS. These observations allowed us to establish that the cells tested for miRNAs expression were indeed consistently modulated by TcES as reported previously by our group [12].

3.2. TcES Do Not Modify Expression of Mir-146 and Mir155 but Downregulate Expression of Let-7i. miRNAs control expression of genes through mRNA degradation or translational suppression [1]. The miRNAs mir-146a, mir-155, and let-7i have been reported to play a role in the induction of maturation and activation of DCs [14-17]. Currently, it is unknown if helminth parasites or their antigens exert a modulatory activity on these host miRNAs. Since in a previous work we found that TcES affects the maturation and secretion of pro-inflammatory cytokines in DCs activated with LPS [12], we explored if these antigens affect the expression of the LPS-induced mir-146a, mir-155, and let-7i in human DCs.

qRT-PCR was performed in independent DCs culture experiments of nine healthy donors $(n=4$ for $3 \mathrm{~h}$ and $n=5$ for $24 \mathrm{~h}$ ) and two reference genes ( $u 6$ and let-7a) were used to establish fold-induction of miRNAs. As already described by others $[18,19]$, we found that at $24 \mathrm{~h}$ after stimulation of DCs, LPS upregulated the expression of mature mir146a, mir-155, and let7i when compared to cells receiving medium alone (Figure 2). Exposure of DCs to TcES alone did not induce an upregulation on the three selected miRNAs (Figure 2) but when these cells were exposed to the combination of TcES plus LPS we observed a contrasting response. Prior to such combined stimuli, we found no significant differences in the expression of mir-146a and mir-155 (Figures 2(a) and 2(b)), indicating that TcES do not modulate the expression of these two miRNAs in human DCs activated by LPS. However, when we assessed the influence of such antigens over the LPS-induced expression of let-7i, the upregulation of this miRNA was no longer observed: statistical difference was found between LPS and LPS/TcES whereas none was found between LPS/TcES and RPMI (Figure 2(c)). At $3 \mathrm{~h}$ after stimulation, the expression of none of the selected miRNAs was statistically significant between groups (Figure 2). Results with the reference gene U6 were found similar to those with let-7a (data not shown). Altogether, these data show that the helminth-derived antigens TcES importantly interfere with the LPS-induced expression of let$7 \mathrm{i}$ in human DCs and suggest that such antigens prevent the upregulation of this miRNA rather that diminishing it after a prior augmentation. TcES appear to exert a fine modulatory activity upon host miRNAs since even when mir-146a and mir-155 have also been related to the LPS-induced response in DCs, only let-7i was affected by such antigens. Thus, this is the first evidence showing that antigens derived from a helminth parasite, indeed, possess modulatory activities over the expression of miRNAs in human DCs.

The lethal-7 (let-7) family of miRNAs has been linked to important aspects of the immune response, such as inflammation, autoimmunity, and host-pathogen interactions [21, 22]. In particular, the expression of its member let-7i seems to be affected by some protozoans like Cryptosporidium parvum and Plasmodium berghei and in turn possibly affecting the immune response against them $[23,24]$. More importantly, let-7i is up-regulated in DCs in response to LPS and participates in the induction of their maturation and secretion of proinflammatory cytokines such as IL-12 [17, 18]. Interestingly, secretion of IL-10 is only favored when let-7i is inhibited [17], indicating the inflammatory activity that this miRNA possesses on DCs activated by LPS. Here we show that TcES downmodulate the LPS-induced expression of let$7 \mathrm{i}$ in human DCs in parallel to a diminished inflammatory cytokine/chemokine profile and a normal secretion of IL10. Thus, it is likely that the previously reported impaired maturation and the repeatedly observed anti-inflammatory phenotype of DCs exposed to TcES can be due to the repressing effects that these antigens possess upon let-7i. Moreover, it has been shown that one of the targets of let$7 \mathrm{i}$ is the suppressor of cytokine signaling 1 (SOCS1) [17], a molecule that has been related to the downregulation of DC maturation and secretion of the pro-inflammatory cytokines IL-12, IL-6, and IFN- $\gamma[25,26]$. Even when we did not assess the expression of SOCS1 in human DCs exposed to TcES, we hypothesize that the downregulation of let-7i by these antigens may in turn affect the levels of SOCS1 regulating in this manner the maturation and the inflammatory cytokine profile of such DCs. The mechanisms involved in the modulation of TcES on let-7i expression in human DCs are currently unknown. Interestingly, O'Hara and colleagues showed that C. parvum parasite decreases let-7i expression by promoting the formation of a NF- $\kappa \mathrm{B}$ p $50-\mathrm{C} / \mathrm{EBP} \beta$ silencer complex in cholangiocytes [27]. If this is the mechanism involved in the repression of let-7i expression by the helminth antigens used in our study remains to be determined.

In conclusion, TcES are helminth-derived antigens that exert modulatory effects upon the expression of the LPSinduced miRNA let-7i. It is possible that this downmodulation underlies the previously reported dampening of maturation in human DCs activated with LPS and the diminished secretion of pro-inflammatory cytokines observed again in this study. To our knowledge, this is the first report that assesses the possible regulatory actions of helminth antigens upon host miRNAs. The downregulation of let-7i by TcES opens a new route in the study of modulation of the host response by $T$. crassiceps and possibly other helminths. This study establishes the basis for the implement of functional studies where silencing and overexpression of miRNAs may allow us to elucidate the complex mechanisms involved in the regulatory activities that helminth antigens possess over the immune system.

\section{Authors' Contribution}

The two authors L. I. Terrazas and F. Sánchez-Muñoz contributed equally to this work.

\section{Acknowledgments}

This work was supported by Instituto Nacional de Cardiología Ignacio Chávez. The authors thank the laboratory 

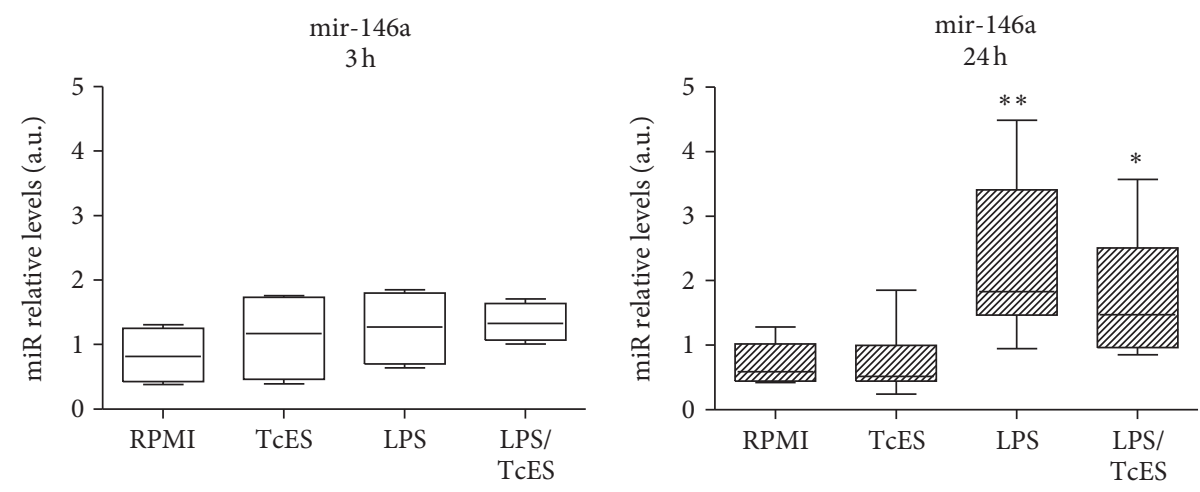

(a)
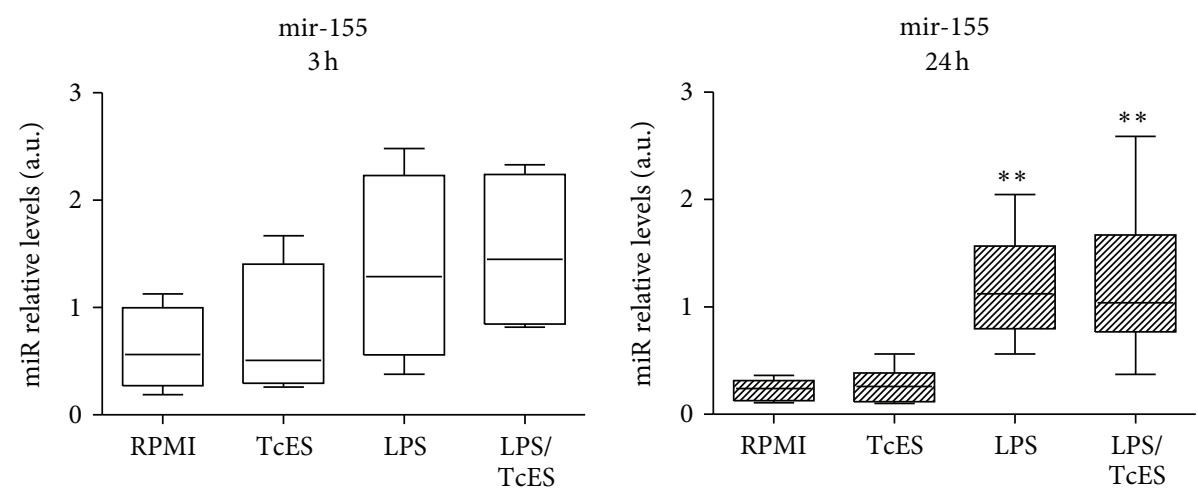

(b)
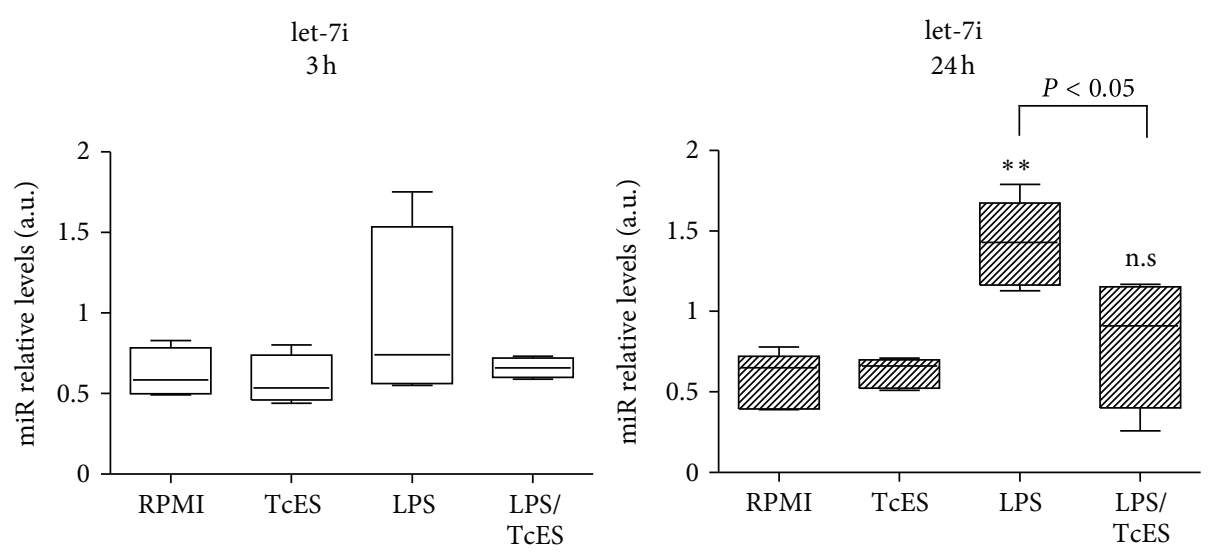

(c)

Figure 2: Expression of miRNAs mir-146a, mir-155, and let-7i in human DCs activated with LPS and exposed to excreted/secreted T. crassiceps antigens. The miRNAs expression was evaluated after stimulation of human DCs with $20 \mathrm{ug} / \mathrm{mL}$ TcES, $1 \mathrm{ug} / \mathrm{mL} \mathrm{LPS}, \mathrm{or}$ a combination of them for 3 or $24 \mathrm{~h}$. Control cells received RPMI. Determination of miRNAs relative levels by qRT-PCR was conducted using mature miRNAs specific TaqMan assays in cultures from nine independent experiments ( 4 donors for $3 \mathrm{~h}$ and $5 \mathrm{donors}$ for $24 \mathrm{~h}$ ). The gene let-7a was used as reference gene and relative quantification was calculated by the formula $2^{-(\mathrm{CT} \text { targetmiRNAs-CT reference) }}$. All miRNAs assays were tested for reproducibility and linearity (PCR efficiency was between 1.9 and 2.0 for all assays). The data is shown as boxplot, horizontal line denotes the median value, box encompasses the upper and lower quartiles and the whiskers, and the minimum and maximum data value. The relative expression values were analyzed using the nonparametric Kruskal-Wallis test and Dunn's post hoc comparisons. For (a) y (b) ${ }^{*} P<0.05$ and ${ }^{* *} P<0.01$ versus RPMI control group were deemed significant. For (c) ${ }^{*} P<0.05$ for LPS versus LPS/TcES and n.s. for LPS/TcES versus RPMI control group. All analyses were performed with the GraphPad Prism v. 5 statistical software. 
technician Angeles Soster Contreras for her assistance to obtain blood samples.

\section{References}

[1] D. P. Bartel, "MicroRNAs: genomics, biogenesis, mechanism, and function," Cell, vol. 116, no. 2, pp. 281-297, 2004.

[2] S. T. Hashimi, J. A. Fulcher, M. H. Chang, L. Gov, S. Wang, and B. Lee, "MicroRNA profiling identifies miR-34a and miR21 and their target genes JAG1 and WNT1 in the coordinate regulation of dendritic cell differentiation," Blood, vol. 114, no. 2, pp. 404-414, 2009.

[3] D. S. Rao, R. M. O'Connell, A. A. Chaudhuri, Y. GarciaFlores, T. L. Geiger, and D. Baltimore, "MicroRNA-34a perturbs B lymphocyte development by repressing the forkhead box transcription factor Foxp 1," Immunity, vol. 33, no. 1, pp. 48-59, 2010.

[4] S. A. Muljo, K. Mark Ansel, C. Kanellopoulou, D. M. Livingston, A. Rao, and K. Rajewsky, "Aberrant T cell differentiation in the absence of Dicer," The Journal of Experimental Medicine, vol. 202, no. 2, pp. 261-269, 2005.

[5] G. Liu, A. Friggeri, Y. Yang, Y. J. Park, Y. Tsuruta, and E. Abraham, "miR-147, a microRNA that is induced upon tolllike receptor stimulation, regulates murine macrophage inflammatory responses," Proceedings of the National Academy of Sciences of the United States of America, vol. 106, no. 37, pp. 15819-15824, 2009.

[6] T. X. Lu, J. Hartner, E.-J. Lim et al., "MicroRNA-21 limits in vivo immune response-mediated activation of the IL-12/IFN$\gamma$ pathway, Th1 polarization, and the severity of delayed-type hypersensitivity," The Journal of Immunology, vol. 187, no. 6, pp. 3362-3373, 2011.

[7] E. Vigorito, K. L. Perks, C. Abreu-Goodger et al., "microRNA155 regulates the generation of immunoglobulin class-switched plasma cells," Immunity, vol. 27, no. 6, pp. 847-859, 2007.

[8] R. M. O'Connell, D. S. Rao, A. A. Chaudhuri, and D. Baltimore, "Physiological and pathological roles for microRNAs in the immune system," Nature Reviews Immunology, vol. 10, no. 2, pp. 111-122, 2010.

[9] C. H. Lecellier, P. Dunoyer, K. Arar et al., "A cellular microRNA mediates antiviral defense in human cells," Science, vol. 308, no. 5721, pp. 557-560, 2005.

[10] G. M. Zeiner, K. L. Norman, J. M. Thomson, S. M. Hammond, and J. C. Boothroyd, "Toxoplasma gondii infection specifically increases the levels of key host microRNAs," PLoS ONE, vol. 5, no. 1, Article ID e8742, 2010.

[11] G. Hu, R. Zhou, J. Liu, A. Y. Gong, and X. M. Chen, "MicroRNA-98 and let-7 regulate expression of suppressor of cytokine signaling 4 in biliary epithelial cells in response to Cryptosporidium parvum infection," Journal of Infectious Diseases, vol. 202, no. 1, pp. 125-135, 2010.

[12] C. A. Terrazas, F. Sánchez-Muñoz, A. M. Mejí-Domínguez et al., "Cestode antigens induce a tolerogenic-like phenotype and inhibit LPS in-flammatory responses in human dendritic cells," International Journal of Biological Sciences, vol. 7, no. 9, pp. 1391-1400, 2011.

[13] B. Pulendran, K. Palucka, and J. Banchereau, "Sensing pathogens and tuning immune responses," Science, vol. 293, no. 5528, pp. 253-256, 2001.

[14] F. Sallusto, B. Palermo, D. Lenig et al., "Distinct patterns and kinetics of chemokine production regulate dendritic cell function," European Journal of Immunology, vol. 29, no. 5, pp. 1617-1625, 1999.

[15] H. B. Mou, M. F. Lin, H. Huang, and Z. Cai, “Transforming growth factor- $\beta 1$ modulates lipopolysaccharide-induced cytokine/chemokine production and inhibits nuclear factor$\kappa \mathrm{B}$, extracellular signal-regulated kinases and p38 activation in dendritic cells in mice," Transplantation Proceedings, vol. 43, no. 5, pp. 2049-2052, 2011.

[16] C. Lu, X. Huang, X. Zhang et al., "miR-221 and miR-155 regulate human dendritic cell development, apoptosis, and IL-12 production through targeting of p27kip1, KPC1, and SOCS-1," Blood, vol. 117, no. 16, pp. 4293-4303, 2011.

[17] M. Zhang, F. Liu, H. Jia et al., "Inhibition of microRNA let$7 \mathrm{i}$ depresses maturation and functional state of dendritic cells in response to lipopolysaccharide stimulation via targeting suppressor of cytokine signaling 1," The Journal of Immunology, vol. 187, no. 4, pp. 1674-1683, 2011.

[18] P. Jin, T. H. Han, J. Ren et al., "Molecular signatures of maturing dendritic cells: implications for testing the quality of dendritic cell therapies," Journal of Translational Medicine, vol. 8, article 4, 2010.

[19] B. J. H. Jansen, I. E. Sama, D. Eleveld-Trancikova et al., "MicroRNA genes preferentially expressed in dendritic cells contain sites for conserved transcription factor binding motifs in their promoters," BMC Genomics, vol. 12, article 330, 2011.

[20] M. Ortiz, F. Schreiber, S. Benitez et al., "Effects of chronic ascariasis and trichuriasis on cytokine production and gene expression in human blood: a cross-sectional study," PLoS Neglected Tropical Diseases, vol. 5, no. 6, Article ID e1157, 2011.

[21] K. M. Pauley, M. Satoh, A. L. Chan, M. R. Bubb, W. H. Reeves, and E. K. L. Chan, "Upregulated miR-146a expression in peripheral blood mononuclear cells from rheumatoid arthritis patients," Arthritis Research and Therapy, vol. 10, no. 4, article R101, 2008.

[22] A. Androulidaki, D. Iliopoulos, A. Arranz et al., "The kinase Akt1 controls macrophage response to lipopolysaccharide by regulating microRNAs," Immunity, vol. 31, no. 2, pp. 220-231, 2009.

[23] X. M. Chen, P. L. Splinter, S. P. O’Hara, and N. F. LaRusso, "A cellular micro-RNA, let-7i, regulates toll-like receptor 4 expression and contributes to cholangiocyte immune responses against Cryptosporidium parvum infection," The Journal of Biological Chemistry, vol. 282, no. 39, pp. 28929-28938, 2007.

[24] F. El-Assaad, C. Hempel, V. Combes et al., "Differential microRNA expression in experimental cerebral and noncerebral malaria," Infection and Immunity, vol. 79, no. 6, pp. 2379-2384, 2011.

[25] J. L. Eyles, D. Metcalf, M. J. Grusby, D. J. Hilton, and R. Starr, "Negative regulation of interleukin-12 signaling by suppressor of cytokine signaling-1," The Journal of Biological Chemistry, vol. 277, no. 46, pp. 43735-43740, 2002.

[26] K. Evel-Kabler, X. T. Song, M. Aldrich, X. F. Huang, and S. Y. Chen, "SOCS1 restricts dendritic cells' ability to break self tolerance and induce antitumor immunity by regulating IL-12 production and signaling," The Journal of Clinical Investigation, vol. 116, no. 1, pp. 90-100, 2006.

[27] S. P. O’Hara, P. L. Splinter, G. B. Gajdos et al., "NF $\kappa$ B p50CCAAT/enhancer-binding protein $\beta(\mathrm{C} / \mathrm{EBP} \beta)$-mediated transcriptional repression of microRNA let-7i following microbial infection," The Journal of Biological Chemistry, vol. 285, no. 1, pp. 216-225, 2010. 

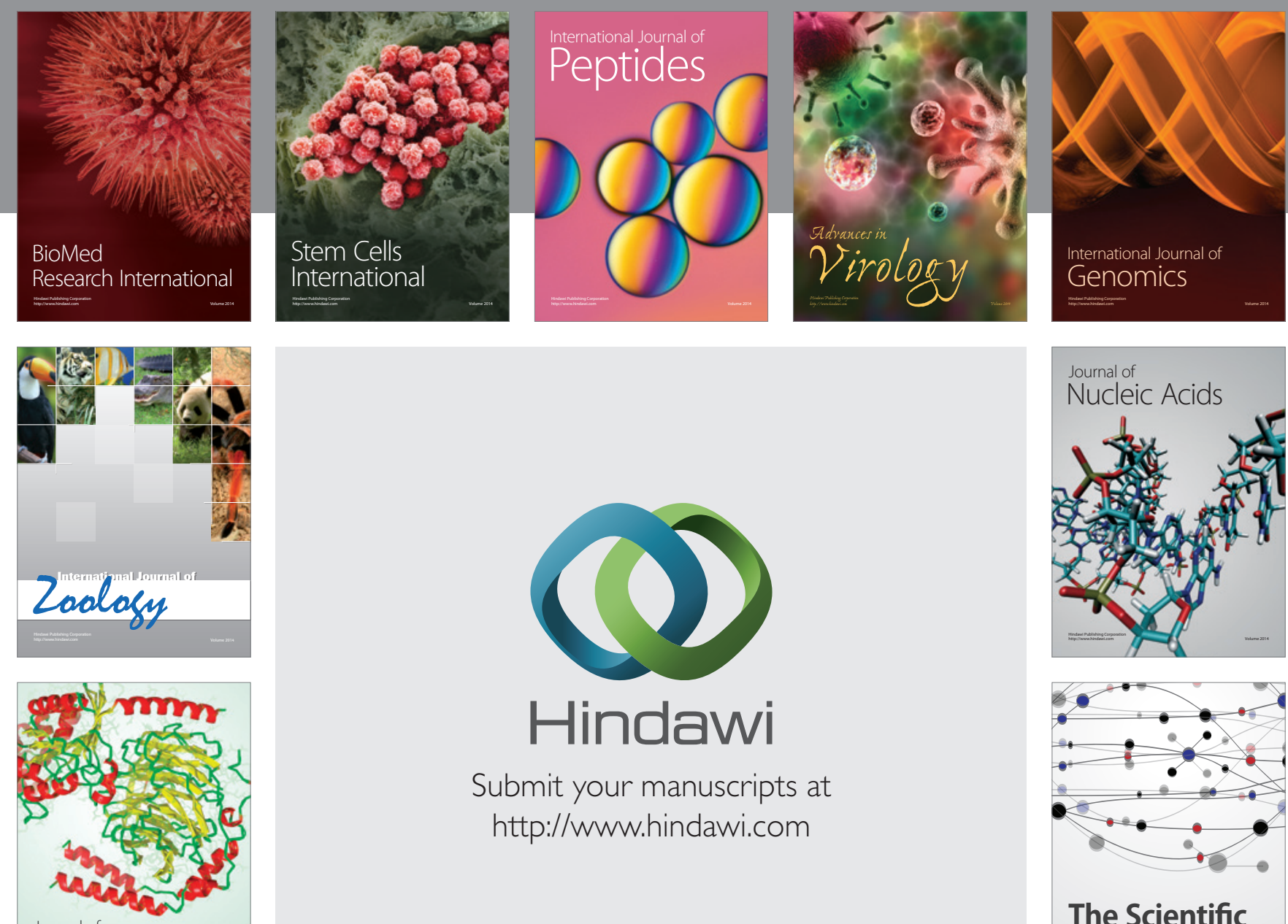

Submit your manuscripts at

http://www.hindawi.com

Journal of
Signal Transduction
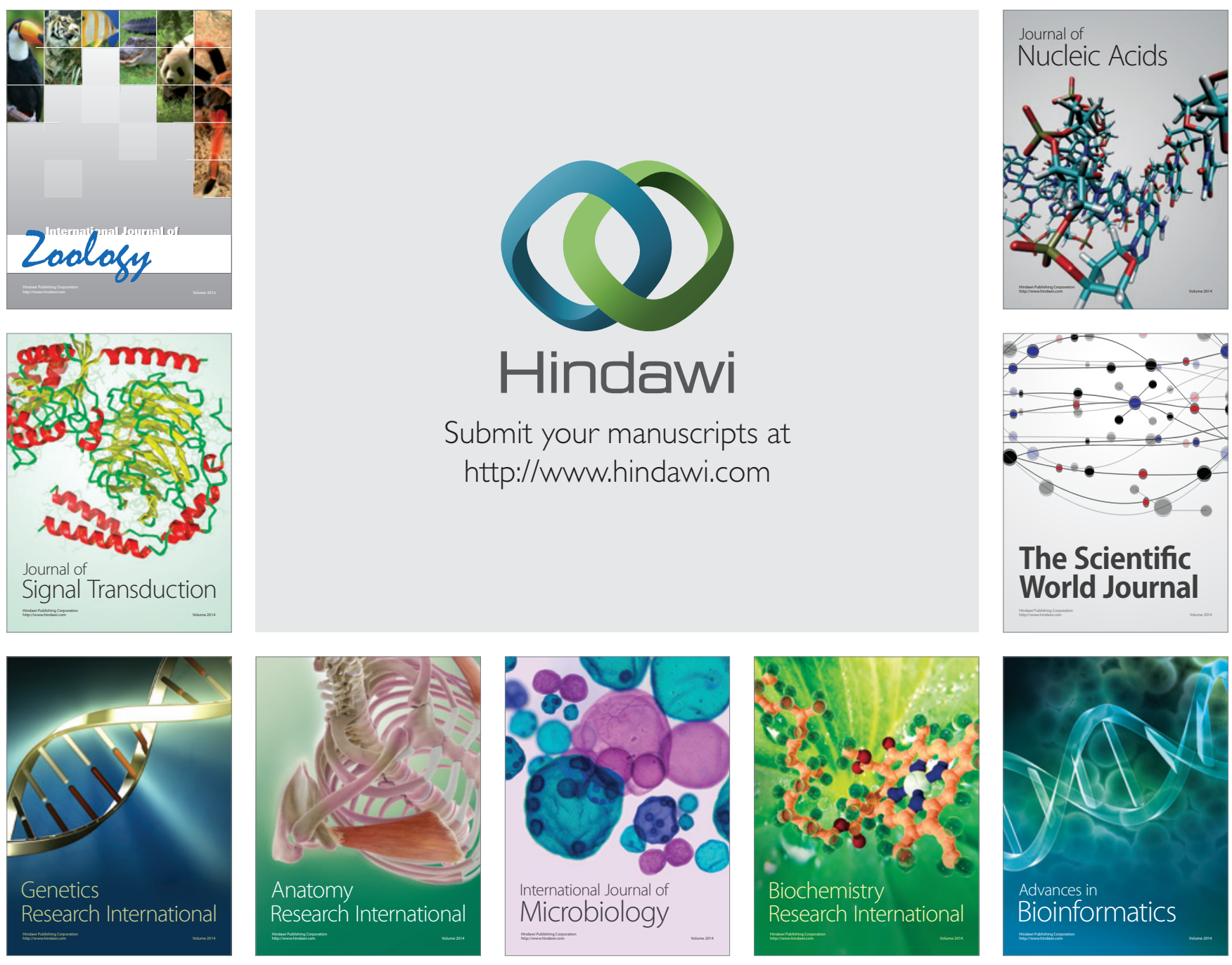

The Scientific World Journal
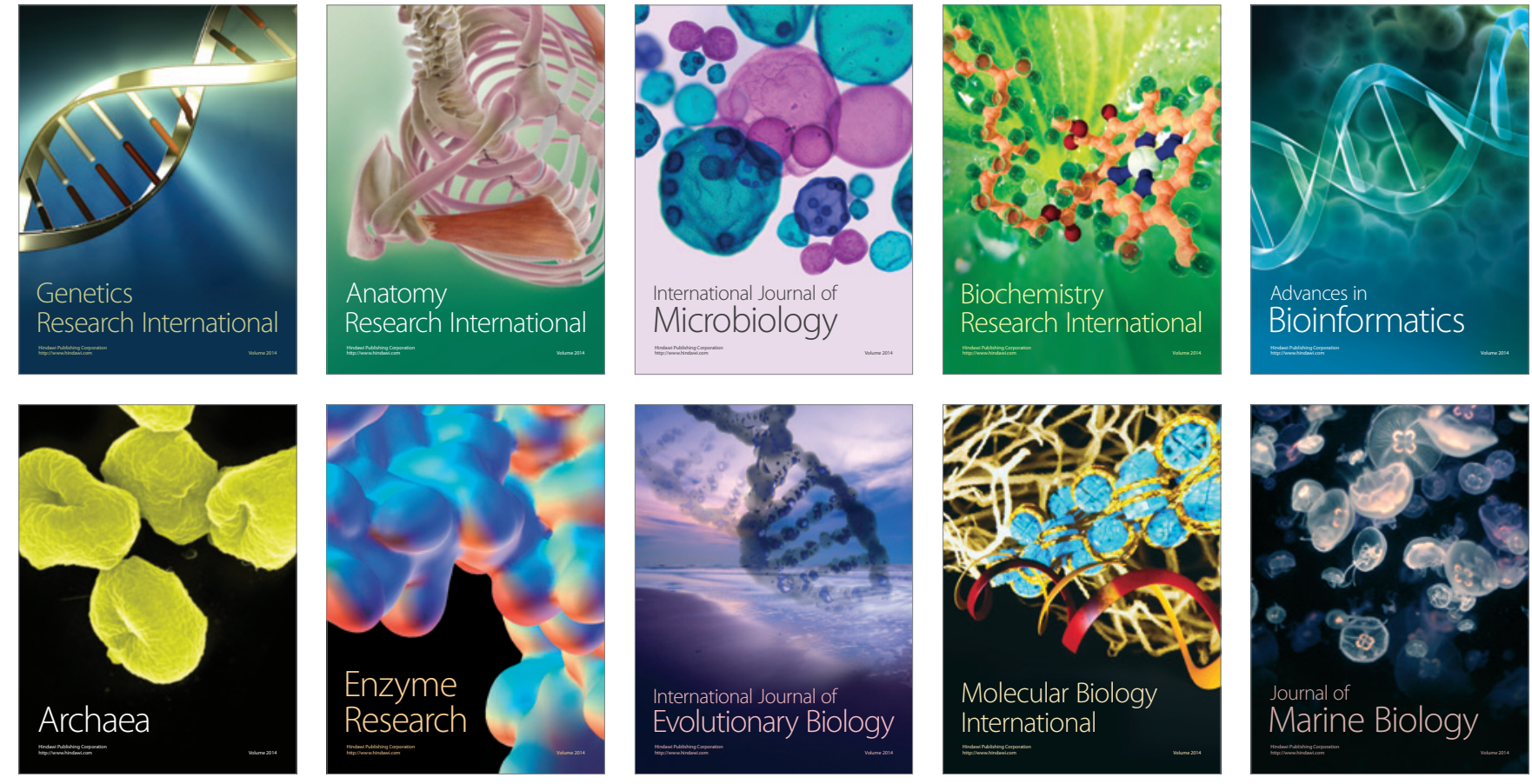